\title{
Type 2 Thanatophoric Dysplasia
}

National Cancer Institute

\section{Source}

National Cancer Institute. Type 2 Thanatophoric Dysplasia. NCI Thesaurus. Code C98584.

Thanatophoric dysplasia characterized by a cloverleaf-like skull and straight femurs. 